\title{
The Afresh Transform Algorithm Based on Limited Histogram Equalization of Low Frequency DCT Coefficients
}

\author{
Hu Jun-peng ${ }^{1}$, Liang Fang ${ }^{2, *}$ and Shen Ji-nan ${ }^{1}$ \\ ${ }^{1}$ School of Information Engineering, Hubei University for Nationalities, Enshi, 445000, China \\ ${ }^{2}$ School of Science, Hubei University for Nationalities, Enshi, 445000, China
}

\begin{abstract}
In reality, face recognition due to illumination, expression, posture, or other objects caused by facial shade seriously affects the recognition rate of the problem, put forward the afresh transform algorithm based on limited histogram equalization of low frequency DCT coefficients. Firstly, divide the image into several non-overlapping local small fragments, and then use limited histogram equalization for local contrast stretching to realize and get rid of the local sub-block noise. Then, eliminate the illumination change in face image by reducing appropriate number of low frequency DCT coefficients. Finally, use kernel principal component analysis for feature extraction, and the nearest neighbor classifier to complete the final face recognition. In ORL, extension Yale B is used in one experiment to verify the effectiveness and the robustness of the proposed algorithm on outdoor facial database. The experimental results show that this algorithm, in dealing with robust face recognition, has achieved higher recognition rate as compared with several linear algorithm.
\end{abstract}

Keywords: Adaptive histogram equalization, Coefficient afresh, Face recognition, The low-frequency discrete cosine transform.

\section{INTRODUCTION}

As a very widely used technology, face recognition [1] has attracted attention of many researchers. It has been widely used in law enforcement, security, voters to verify and buildings, door, car, or network access, and other fields. The performance of face recognition system has reached a satisfactory level [2] under the constraint environment, but its performance is often affected because of illumination, expression, posture, shelter's influence of such conditions reduced greatly [3], as a result, these changes are necessary for automatic face recognition of standardization [4].

Scholars also proposed the method based on local characteristics, where face image was divided into several local blocks, handle each block independently, finally to make decisions based on all the pieces of mixed classification results [5]. For example, literature [6] proposed face sub-space reconstruction algorithm based on robust principal component analysis; literature [7] proposed joint sparse representation algorithm based on low-rank sub-space restore; literature [8] proposed sparse representation of reconstruction algorithm; and literature [9] proposed synergy representation based on the classification. This approach requires that everyone has enough sample to form a probe image (namely query face image), but the actual situation is face often affected by light and so on, leading to low recognition rate. So these methods in real environment are not always feasible. Also, some scholars will first keep out

*Address correspondence to this author at the School of Science, Hubei University of Nationalities, Xueyuan Road 39\#, Enshi, Hubei, 445000, P.R. China; Tel: 18671863389; E-mail: maple liang@163.com of the image divided into sub-block, the extracted features in the sub-block, and then execution of the similarity matching process based on each block in pairs, in order to reduce the matching error caused by keeping out of blocks training produces different afresh value assigned to different blocks. For example, literature [10] proposed local matching face recognition algorithms. Literature [11] proposed partial similarity algorithm. However, the process of training is usually data dependence. Literature [12] showed that the conversion to Discrete Cosine Transform (Discrete Cosine Transform, DCT) of the low frequency coefficient in all other photo-metric normalization technique can get the best performance. Literature [13] using kernel function can effectively calculate the high-dimensional feature space of the principal components. Literature [14] pointed out that the Linear Discriminant Analysis (Linear Discriminant Analysis, LDA) method aims to find a class particular linear projection, the purpose is to maximize the projection image between the scattering matrix and scattering matrix within the class ratio, but it needs the training time than Principal Component Analysis (Principal Component Analysis, PCA), because it uses PCA as processing steps.

The basis of this research is to improve the method of reducing the effects of illumination change to face recognition, and better solve the problem of robust face recognition, by proposing the afresh transform algorithm based on limited histogram equalization of low frequency DCT coefficients. It uses Kernel PCA (Kernel Principal Component Analysis, KPCA) to extract high dimensional feature vector, and uses the nearest neighbor classifier to complete human face recognition by the DCT specification or compensation illumination change. 


\section{THE SYSTEM DESIGN}

In order to improve the recognition rate of face recognition system under the condition of illumination change, face recognition system architecture is proposed, as shown in Fig. (1). The algorithm process is as follows:

1) First take training and testing images order divided into a series of continuously even without overlapping subblock of, after dealing with the CLAHE, image gray level difference increased, contrast enhancement, more details are divided, and contain reduced noise accordingly;

2) By taking every sub-block for DCT after dealing with the CLAHE corresponding coefficient matrix can be obtained. Then, by using standard luminance quantization matrix to uniform quantization of each coefficient, the coefficient matrix can be obtained after quantization. The coefficient matrix is obtained which is divided into two parts for direct current coefficient DC and alternating current coefficient $\mathrm{AC}$, which can be seen from the DCT. The energy is concentrated in a few of the low frequency coefficients, and then through to transform to reduce low frequency DCT coefficient to eliminate the influence of illumination change;

3) Get normalized image (namely free illumination change), using KPCA extracting image features, KPCA can extract the high order statistics, including nuclear function using Gaussian kernel;

4) Finally, using the nearest neighbor classifier to extract the characteristics of the classification and recognition.

The detailed design of each part of the systemis as follows:

\subsection{Contrast Limited Adaptive Histogram Equalization}

Histogram of the image refers to the intensity distribution of image, histogram equalization even adjusts the image's intensity values, and enhances the global contrast of image. This is a very useful pre-processing step, and strengthens the standardization of the given image, however, interest in features within the image needs local reinforcement, so if the standardization of face image is required, it should be local enhancement contrast.
Therefore, the system does not use traditional histogram equalization, but instead use contrast limited adaptive histogram equalization (CLAHE) [15], limited histogram equalization in the image of a small scale operation, known as the tiles. The method is an improved method for $\mathrm{HE}$ algorithm, combined with the limited contrast and adaptive histogram equalization's advantages of two technologies, while enhancing the image contrast can effectively suppress noise interference, better highlight the image detail, and the implementation process is not complicated. Therefore, the system design uses CLAHE technology to enhance the image's contrast.

CLAHE image pre-processing has main implementation steps as follows:

1) Divide into block: take the original image divided into $M \times N$ continuously even without overlapping sub area; The greater for the sub region, the more obvious for the contrast enhancement effect, but the more loss for image detail and edge component. Therefore, this article adopting $8 \times 8$ continuously without overlapping area is relatively reasonable.

2) Calculate each sub-domain gray-level histogram: each histogram record $i$, possibly appearing gray series for $N_{\text {Gray }}$, then each sub-domain corresponding histogram function is expressed by $H(i), 0 \leq i \leq N_{\text {Gray }}-1$.

3) Take each sub-area for gray-scale histograms "shear"; it will be beyond the pixel average distribution of the shear limit amplitude to the other gray-scale.

4) Take each sub-domain's gray-level histogram for equalization processing after contrast limited.

5) Get the center of each area; these points will serve as the sample points.

6) Then by taking each pixel of the image and using formula (1) for Grey linear interpolation processing, get the new gray scale as follows:

$$
M(x, y)=p\left[q I_{--}+(1-q) I_{+-}\right]+(1-p)\left[q I_{-+}+(1-q) I_{++}\right]
$$

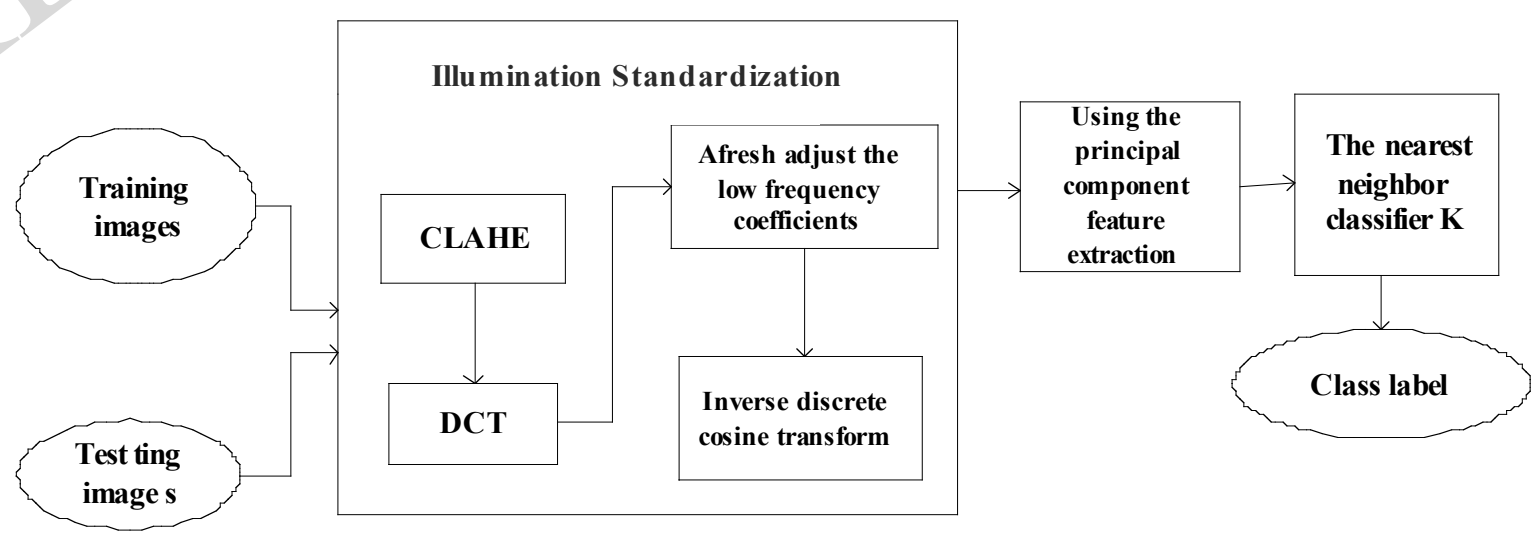

Fig. (1): The design of system architecture. 
Among them:

$p=\frac{y-y_{-}}{y_{+}-y_{-}} q=\frac{x-x_{-}}{x_{+}-x_{-}}$

$M(x, y)$ shows the point $(x, y)$ place Grey value, $I_{--}$ shows evaluation points left sample points $x_{-}, y_{-}$'s Grey value. In the same way, $I_{-+}, I_{+-}, I_{++}$show the other three points bearing Grey value of sample point, respectively.

Fig 2 (a) shows the original image. Fig 2(b) shows image after histogram equalization. Fig 2(c) shows image after contrast limited adaptive histogram equalization.

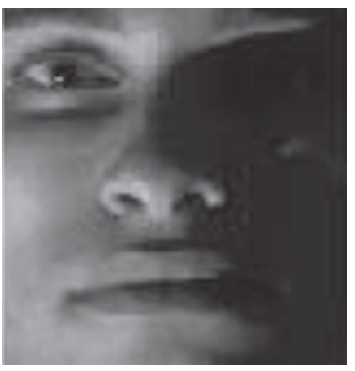

(a) Original image

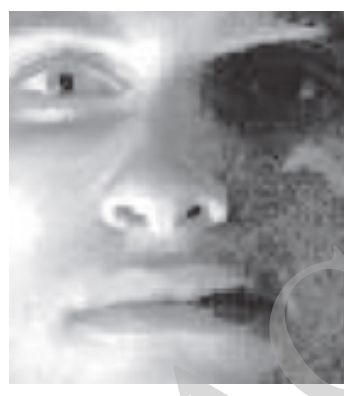

(b) Image after histogram equalization

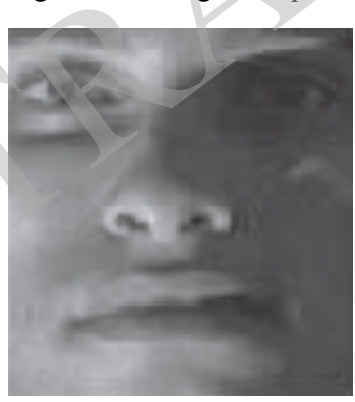

(c) Image after contrast limited adaptive histogram equalization

Fig. (2): Using HE and CLAHE method's contrast figure.

\subsection{Discrete Cosine Transform}

In the DCT, with more than a series of limited data points to represent the cosine function concussion sum under different frequency, the DCT has four types, as follows: DCT I, DCT II, DCT III and DCT IV. DCT II is mostly used in signal processing, usually referred to as DCT. 2 D M X N DCT are defined as follows:

$$
C(u, v)=\alpha(u) \alpha(v) \sum_{x=0}^{M-1} \sum_{y=0}^{N-1} f(x, y) \times \cos \left[\frac{\pi(2 x+1) u}{2 M}\right] \cos \left[\frac{\pi(2 y+1) v}{2 N}\right]
$$

Among them: $u=0,1,2, \cdots, M-1 ; \quad v=0,1,2, \cdots, N-1$, is called the frequency domain transformation factor; $C(u, v)$ shows transform result, also known as DCT coefficients. $\alpha(u), \alpha(v)$ respectively defined as:

$\alpha(u)=\left\{\begin{array}{c}\frac{1}{\sqrt{M}}, \text { if } u=0 \\ \sqrt{\frac{2}{M}}, \text { if } u=1,2, \ldots, M-1\end{array}\right.$

$\alpha(v)=\left\{\begin{array}{c}\frac{1}{\sqrt{N}}, \text { if } v=0 \\ \sqrt{\frac{2}{N}}, \text { if } v=1,2, \ldots, N-1\end{array}\right.$

The corresponding 2D discrete cosine transformation (IDCT) is given by the formula:

$$
\begin{aligned}
& f(x, y)=\sum_{u=0}^{M-1} \sum_{v=0}^{N-1} \alpha(u) \alpha(v) C(u, v) \\
& \times \cos \left[\frac{\pi(2 x+1) u}{2 M}\right] \cos \left[\frac{\pi(2 y+1) v}{2 N}\right]
\end{aligned}
$$

Among them, $x=0,1,2, \cdots, M-1 ; y=0,1,2, \cdots, N-1$

In the designed system, the DCT application on the whole face image converts it as face image's frequency components. As the low frequency DCT is associated with light changes, the illumination change through reduced to compensate for low frequency DCT coefficient reduces the number of DCT coefficients and factor is obtained by experiment. As shown in Fig. (3), different number of low frequency DCT coefficients give different afresh transform's DCT standardization results.

\subsection{Kernel Principal Component Analysis (KPCA)}

Feature extraction is one of the main steps of face recognition system, because it can reduce facial image dimension. The most popular feature extraction technology is Principal Component Analysis (PCA), but the representation of PCA technology is based on image set of the second order statistics. This method cannot show that the image depends on high-order statistics, and the image of higher-order statistics, including pixel intensity value of nonlinear relationship, contains the identification of important information. This system uses Kernel Principal Component Analysis (KPCA) to extract the higher-order statistics, the algorithm process description is as follows:

The first step is to focus on given $n$ samples set of $x_{k}$, among them, $x_{k}=\left[x_{k 1}, x_{k 2}, \ldots, x_{k n}\right]^{T} \in R^{n}$. The PCA is designed to find the maximum variance's projection of $C$, that is the characteristic values are obtained from the covariance matrix, and the problem turns into solving formula (7) characteristic values: 
$\lambda v=C_{x} v$

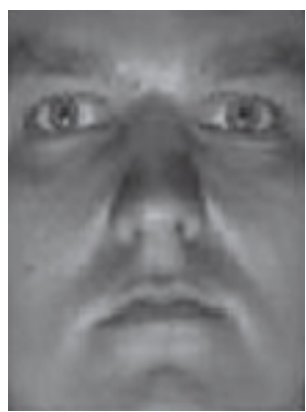

(a) Original image

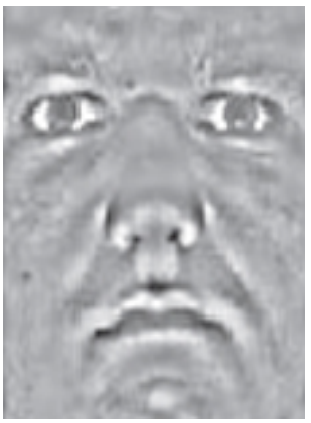

(b) Afresh transform coefficient $=20$

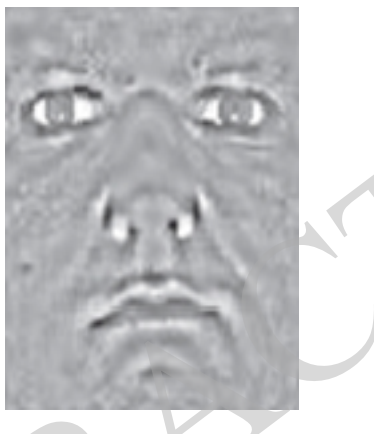

(c) Afresh transform coefficient $=30$

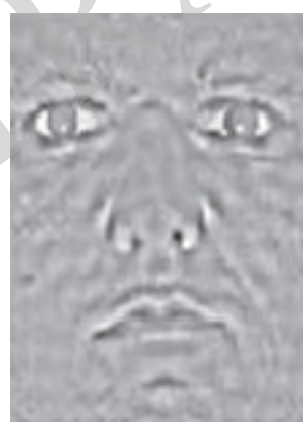

(d) Afresh transform coefficient $=27$

Fig. (3): Different afresh transform coefficient contrast figures.

The above formula, $\lambda \geq 0$ is the characteristic value, $v \in R^{n}$ is the feature vector.

In the kernel PCA, the input space of each vector is projected in a high dimensional feature space $R^{f}$ by nonlinear mapping function of $\varphi: R^{n}-R^{f}$. Therefore, the characteristic value of the kernel PCA can be calculated as follows:

$$
\lambda v_{\phi}=C_{\phi(x)} v_{\phi}=\left(\frac{1}{N} \sum_{i=1}^{N} \phi\left(x_{i}\right) \phi\left(x_{j}\right)\right) v_{\phi}=\frac{1}{N} \sum_{i=1}^{N}\left(\phi\left(x_{i}\right), v_{\phi}\right) \phi\left(x_{j}\right)
$$

$v_{\phi}$ of all $\lambda \neq 0$ solutions within $\phi\left(x_{1}\right), \ldots, \phi\left(x_{n}\right)$ scope, there is factor $\alpha_{i}$, such as:

$v_{\phi}=\sum_{i=1}^{n} \alpha_{i} \phi\left(x_{i}\right)$

Define $N \times N$ kernel matrix $K$, then

$K_{i, j}=\phi\left(x_{i}\right) \cdot \phi\left(x_{j}\right)$

Taking the formula (8) multiplied by $\phi\left(x_{j}\right)$, and putting into the formula (9), we will get:

$N \lambda K \alpha=K^{2 \alpha}$

The solution of characteristic value problem can be expressed as more simple characteristic value problem:

$N \lambda \alpha=K \alpha$

Feature space's projection is carried out by the following formula:

$$
\varphi(x)_{K P C}^{k}=v_{\varphi}^{k} \cdot \varphi(x)=\sum_{i=1}^{N} \alpha_{i}^{k}\left(\varphi\left(x_{i}\right) \varphi\left(x_{j}\right)\right)=\sum \alpha_{i}^{k} K\left(x_{i}, x_{j}\right)
$$

Therefore, it does not need a large amount of calculation, and can extract the $k$ nonlinear principal components by using the kernel function, and select Gaussian kernel function $k(x, y)=\exp \left(-\gamma\|x-y\|^{2}\right)$, because it has superior performance in many pattern classification applications.

\subsection{The Nearest Neighbor Classification}

Classification is a very crucial step in any face recognition system. The k- nearest neighbor classifier is the simplest and the most widely used non parametric classifier. The objects are classified based on the feature space of the most nearest classifier to the training instances. Initially, feature extraction is completed; the system is calculated between the test sample and each training sample characteristics of the Euclidean distance; and then by using the nearest neighbor classifier, the final classification and recognition is completed.

\section{THE EXPERIMENTAL RESULTS AND ANALYSIS}

Experiments are implemented on a personal computer by using MATLAB 7.0. The computer is configured as: Windows XP operating system, CENTRINO COREII processor, the main frequency $2.53 \mathrm{GHz}, 4 \mathrm{~GB}$ of RAM.

It has performed a large number of experiments in a public database ORL standard face's library, extension Yale $\mathrm{B}$ and an outdoor face database [15]. The algorithm used in 
this study selected the impact of face recognition rate and recognition speed based on the different number of DCT coefficients in the ORL face database. It tests the algorithm's robustness to illumination change by using expanded Yale B database. The outdoor face database contains the uncontrolled street light's positive photograph of unfamiliar face images. Keeping out sources including glasses, hats, hair and hands on the face, in addition to shelter, the image changes also include expression changes and posture changes.

\subsection{The Afresh Transform DCT Coefficients}

The experiment was conducted on ORL face database, ORL face image database including 400 face images, total of 40 people, which include everyone at different times, different illuminations, different head angles, different facial expressions and different facial details condition of 10 human face images. Every image gray levels for 256, resolution for all was set at $112 \times 92$ pixels. Experimental comparison of the effect of the performance of this paper's algorithm by the choice of different number of DCT coefficients was done, including the recognition accuracy and recognition speed (the whole algorithm average consuming time), afresh adjustment of the number of DCT coefficients, respectively taking 7, 13, 19, 27, 37 and 47. Fig. (4) shows the impact curve based on the recognition accuracy and recognition speed by the different coefficient numbers. From Fig. (4), you can see that with the increase of number of DCT coefficients, the identification accuracy also increases, but as the number of coefficient exceeds a certain extent, the curve shows that further increasing the coefficient plays no significant role in the promotion of recognition rate, and shows coefficient of deleted image reconstruction and the influence of face recognition are very faint. Moreover, the increasing coefficient influences recognition time drastically. The higher the coefficient, the greater the time overhead of calculation, and longer the system identification need. Therefore, the experiments have proved that in order to obtain ideal recognition rate and recognition speed, select the appropriate number of DCT coefficients. This paper's algorithm is based on the system to achieve ideal recognition rate, and at the same time satisfies the requirement of realtime face recognition algorithm to identify speed increase as much as possible to determine the appropriate number of DCT coefficients, through the analysis (Fig. 4) of different number of coefficients of influence curve for recognition rate and recognition speed. This article suggests that 27 DCT coefficient obtained recognition effect is the best, the number of DCT coefficients proportional to the recognition rate was not obtained, and also suggests that using a very high DCT coefficient does not necessarily obtain the best recognition rate but increase the operation time overhead. Therefore, in the following experiments, afresh transform DCT coefficient number is taken as 27.

\subsection{The Canonical Analysis}

Extension of the Yale B database [3] contains 38 objects in 64 images under the condition of different illuminations. Each object under different light conditions has five subsets.
Subset 1 contains everyone's 7 images, subset of 2, 3, 4, and 5 respectively contain $12,12,14$, and 19 images. The greater the number of subsets, the more obvious the illumination change. Experiment takes all images as the unified clipping for $64 \times 64$ size, as shown in Fig. (5) for extension of Yale B face image sample after clipping on face database.
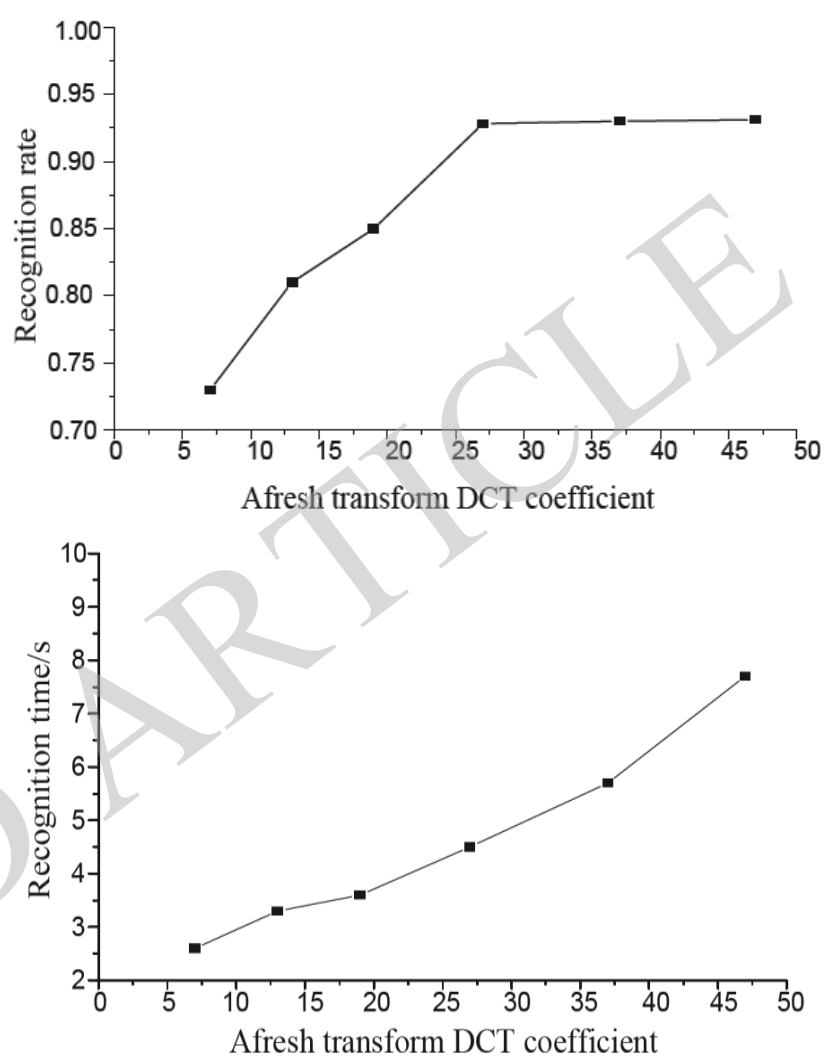

Fig. (4): Different coefficient numbers of the impact curve based on the recognition accuracy and recognition speed.

In the experiment, subset 1 is used for training, because it is closer to the positive illumination. Subsets of 2, 3, 4 and 5 are used for testing. The number of DCT coefficient was set at 27. KPCA on the number of principal components is set for 50 , Gaussian kernel function $k(x, y)$ parameter is set for $\gamma=2^{(n-20) / 2.5} \gamma_{0}, n=0,1, \ldots, 20$, among of them, $\gamma_{0}$ shows training set of standard deviation. In addition to testing algorithm of this paper, experiment by three kinds of techniques, respectively for the "no illumination normalization", "only using the histogram equalization", "using afresh adjust the low frequency DCT coefficient and histogram equalization" was also conducted, as shown in Table 1 for several standardization technology on the extension of Yale B database's recognition results.

It can be observed from Table $\mathbf{1}$ that recognition rate of the subset 5 is $6.5097 \%$, which is very low. It can also be observed that the recognition of the test set is actually quite difficult. By using the IN technology, such as histogram equalization, recognition rate up to $38.0803 \%$, the recognition rate is also challenging. By using the afresh transform low frequency DCT coefficient including the HE, 


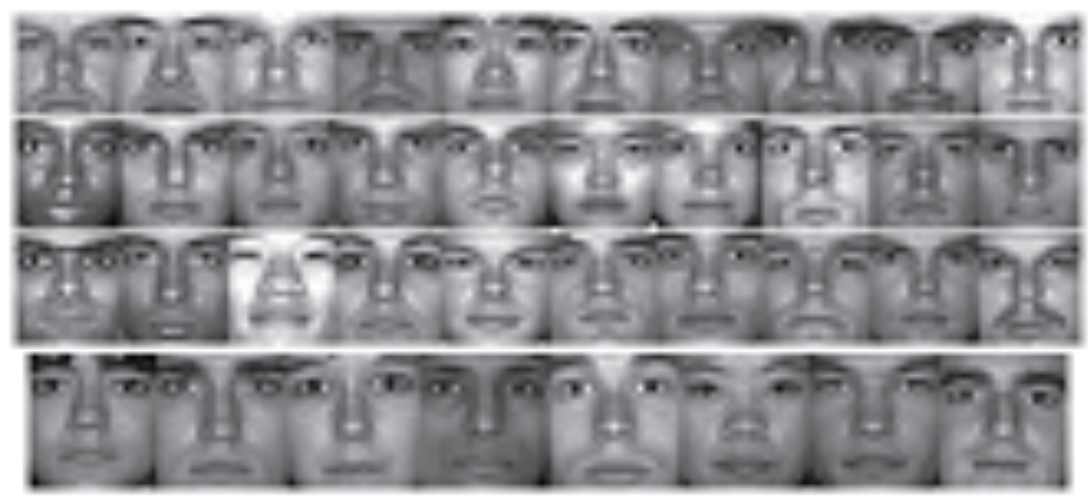

Fig. (5): Extension the Yale B database of face image sample after cutting.

Table 1: Different illumination standardization technology recognition rates (\%).

\begin{tabular}{|c|c|c|c|c|}
\hline Using Technology & Subset 2 & Subset 3 & Subset 4 & Subset 5 \\
\hline Afresh adjust the low frequency DCT coefficients and histogram equalization & 100 & 100 & 89.0923 & 94.7632 \\
\hline $\begin{array}{l}\text { This paper's algorithm (CLAHE and afresh transform DCT coefficient of low } \\
\text { frequency) }\end{array}$ & & 100 & 97.5564 & 98.1609 \\
\hline
\end{tabular}

the recognition rate improved to $94.7632 \%$, showing that the method has great improvement ability. By the use of the proposed CLAHE combined with DCT for illumination normalization, recognition rate of the subset 5 can be as high as $98.1609 \%$.

The proposed standardization technique gives the best recognition rate of $100 \%$ based on the subset 2 and the subset 3. Recognition rate of the subset 4 is $97.5564 \%$, by using CLAHE combined with DCT, recognition rate of the subset 5 increased from $6.5097 \%$ to $98.1609 \%$, which is a significant improvement, showing the effectiveness of this paper's algorithm.

\subsection{Illumination Change Face Recognition}

In order to demonstrate the superiority of this paper's algorithm, the recognition rates of this algorithm and other several relatively advanced algorithms are compared, including the logarithmic transformation [2], gamma ray intensity calibration [4], homomorphic filtering [8], single scale Retinex [7], wavelet standardization [9], and wavelet denouncing [10]. The parameters set of each comparison algorithm corresponds respectively with reference to each algorithm in the literature. Experiment used subset 1 as the training set, the remaining subsets 2 to 5 for testing, and identification results are shown in Table 2.

It can be observed from Table 2 that for all the subsets, the recognition rate of this paper's algorithm is higher than all other algorithms. All algorithms in the subset 2 and the subset 3 have high recognition rates, so it cannot clearly demonstrate the superiority of this paper's algorithm. It is very difficult to identify the subset 4 and the subset 5 , as the recognition rate of this paper's algorithm remained above

Table 2: Each algorithm recognition rate based on expands in Yale B (\%).

\begin{tabular}{|c|c|c|c|c|}
\hline Using Technology & Subset 2 & Subset 3 & Subset 4 & Subset 5 \\
\hline \hline Logarithmic transformation & 93.4909 & 93.3596 & 43.5789 & 31.6150 \\
\hline Gamma ray intensity correction & 97.3901 & 97.6316 & 43.5789 & 38.2188 \\
\hline Homomorphic filtering & 97.7892 & 97.3316 & 80.3609 & 91.4586 \\
\hline Single scale Retinex & 99.6084 & 99.4597 & 93.5429 \\
\hline Wavelet standardization & 100 & 100 & 93.3910 & 96.0942 \\
\hline Wavelet denoising & 99.8002 & 99.7193 & 97.5564 & 98.1609 \\
\hline This paper's algorithm & 100 & 100 & & 9 \\
\hline
\end{tabular}


Table 3: The recognition rate of outdoor face image $(\%)$.

\begin{tabular}{|c|c|c|c|c|}
\hline Logarithmic transformation & 56.4 & 66.4 & 70.9 & 71.8 \\
\hline Homomorphic filtering & 40.5 & 47.4 & 57.0 & 67.1 \\
\hline Wavelet denoising & 39.2 & 45.0 & 53.8 & 68.2 \\
\hline This paper's algorithm & 61.8 & 76.4 & 77.3 & 81.8 \\
\hline
\end{tabular}

Table 4:Each algorithm of the running time comparison based on the extended Yale $B$.

\begin{tabular}{|c|c|c|}
\hline Algorithm & Training the Total Completion Time (second) & Test Image Time (second) \\
\hline \hline Logarithmic transformation & 159.9829 & 3.7821 \\
\hline Gamma ray intensity correction & 184.0392 & 4.8729 \\
\hline Homomorphic filtering & 130.2983 & 4.0921 \\
\hline Single scale Retinex & 100.0772 & 4.7011 \\
\hline Wavelet standardization & 98.7829 & 3.9804 \\
\hline Wavelet denoising & 99.0881 & 3.7849 \\
\hline This paper's algorithm & 98.7961 & 2.8941 \\
\hline
\end{tabular}

$97 \%$ and $98 \%$, and the logarithmic transformation, gamma rays intensity correction, homomorphic filtering recognition rate below $50 \%$, even as low as $31.6150 \%$, which reflect the reliability of this paper's algorithm in terms of recognition rate and robustness of illumination change.

It can be observed from Table 3 that due to the challenge of natural barrier, all the algorithms have relatively low overall recognition rate. Examples of the challenging images are shown in Fig. (6). We can see that compared with other algorithms, this paper's algorithm can still get the best recognition performance, thus signifying its reliability and strong robustness.
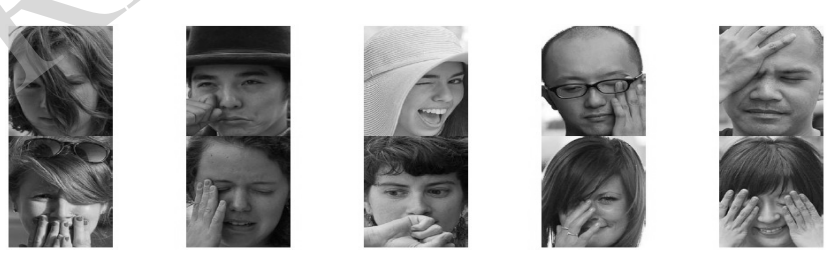

Fig. (6): The image sample of the outdoor face database.

\subsection{Performance Comparison}

Only recognition rate being higher than other algorithms is not enough to illustrate the superiority of this paper's algorithm, therefore, the experiment recorded the operation time of each algorithm, including training total completion time, and testing one image time. The results are shown in Table 4.
It can be observed from Table 4 that the training total completion time of this paper's algorithm is only 0.0132 seconds higher than wavelet standardization, which is a lot less than several other comparison algorithms at timeconsuming. Test one image only needs 2.8941 seconds, significantly lower than all other algorithms, which indicates the superiority of this paper's algorithm.

\section{CONCLUSION}

In order to improve face recognition accuracy under the condition of "bad", and improve the robustness of the algorithm, this paper proposed the afresh transform algorithm based on limited histogram equalization of low frequency DCT coefficients. The study used the CLAHE technology instead of the HE technology for comparing stretched, namely in smaller called tiles on the area of operation to replace the operation on the whole face image. And then eliminated illumination changes by the afresh transform to reduce low frequency DCT coefficient, using kernel PCA to extract the image of high order statistics, and using the nearest neighbor classifier classification. It is very difficult to give the best recognition rate as high as $98.1609 \%$ based on the identification of extension of the Yale B's subset 5. On comparison with several advanced algorithms, this algorithm showed to be effective and robust.

This paper's algorithm will be applied to other face data sets in the future, combined with other advanced 
technologies, having a large number of experiments, improving the recognition rate at the same time, and further reducing the running time of this algorithm, in order to better apply to the real-time robust face recognition system.

\section{CONFLICT OF INTEREST}

The authors confirm that this article content has no conflict of interest.

\section{ACKNOWLEDGEMENTS}

None declared.

\section{REFERENCES}

[1] Y. Su, S.G. Shan, X.L. Chen, and W. Gao, "Integration of globa and local feature for face recognition," Journal of Software, vol. 21, no. 8, pp. 1849-1862, 2010.

[2] Y. Tian, Y. Yun, and T. Ma, "Discriminant neighborhood preserving embedding algorithm for face recognition," Journal of Xidian University, vol. 38, no. 3, pp. 24-28, 2011.

[3] X. Li, and F.P. Da, "A rapid method for 3D face recognition based on rejection algorithm," Acta Automatica Sinica, vol. 36, no. 1, pp. $153-158,2010$.

[4] X. Li, and D.A. Fei-Peng, "3D face recognition based on profile and rigid regions," Journal of Image and Graphics, vol. 15, no. 2, pp. 266-273, 2010.

[5] H. Yin, P. Fu, and X. Sha, "Face recognition based on DCT and LDA," Acta Electronica Sinica, vol. 37, no. 10, pp. 2211-2214, 2009.
[6] W. Ge, G. Li, Y. Cheng, C. Xue and M. Zhu, "Face image illumination processing based on improved Retinex," Optics and Precision Engineering, vol. 18, no. 4, pp. 1011-1020, 2010.

[7] J. Sun, Y. Li, X. Meng, and J. Liu, "Improved monoscale Retinex and application in face recognition," Application Research of Computers, vol. 28, no. 12, pp. 4790-4793, 2011.

[8] Z. Jiao, and B. Xu, "Color image illumination compensation based on homomorphic filtering," Journal of Optoelectronics Laser, vol. 21 , no. 4, pp. 602-605, 2010

[9] T. Zhang, B. Fang, Y. Yuan, Y. Y. Tang, Z. Shang, D. Li, and F. Lang, "Multiscale facial structure representation for face recognition under varying illumination," Pattern Recognition, vol. 42, no. 2, pp. 251-258, 2009.

[10] Y. Li, L. Meng, and J. Feng, Lighting Coefficients Transfer Based Face Illumination Normalization, Pattern Recognition, Springer: Berlin Heidelberg, 2012, pp. 268-275.

[11] J. Li, B. Zhao, H. Zhang and J. Jiao, "Fusing DCT and LBP features for face recognition", Transactions of Beijing Institute of Technology, vol. 30, no. 11, pp. 1355-1359, 2010.

[12] V.P. Vishwakarma, S. Pandey, and M.N. Gupta, "An illumination invariant accurate face recognition with down scaling of DCT coefficients," Journal of Computing and Information Technology, vol. 18, no. 1, pp. 53-67, 2010.

[13] G.F. Lu, Z. Lin, and Z. Jin, "Optimal discriminant analysis based on kernel extension of graph embedding and face recognition," Journal of Software, vol. 22, no. 7, pp. 1561-1570, 2011.

[14] J.F. Zou, G. Y. Wang, and X. Gong, "Face recognition based on enhanced gabor feature and direct fractional-step linear discriminant analysis," Pattern Recognition and Artificial Intelligence, vol. 23, no. 4, pp. 477-482, 2010.

[15] T. Celik, and T. Tjahjadi, "Automatic image equalization and contrast enhancement using Gaussian mixture modeling," IEEE Transactions on Image Processings, vol. 21, no. 1, pp. 145-156, 2012.

Received: September 16, 2014 